2020-07-05

\title{
Nonlinear bending of cylindrical shells subjected to transverse loads
}

\author{
ZHU, J
}

http://hdl.handle.net/10026.1/15840

10.1016/j.mechrescom.2020.103561

Mechanics Research Communications

Elsevier BV

All content in PEARL is protected by copyright law. Author manuscripts are made available in accordance with publisher policies. Please cite only the published version using the details provided on the item record or document. In the absence of an open licence (e.g. Creative Commons), permissions for further reuse of content should be sought from the publisher or author. 


\begin{tabular}{|l|c|}
\hline \multicolumn{1}{|c|}{$\begin{array}{c}\text { Mechanics Research Communications. } \\
\text { Year 2020 }\end{array}$} & $\begin{array}{c}\text { Publication Office: } \\
\text { Elsevier UK }\end{array}$ \\
\hline $\begin{array}{l}\text { Editor-in-Chief: A. Rosato New Jersey Institute of Technology, Newark, New Jersey, } \\
\text { USA Anthony.Rosato@ njit.edu }\end{array}$ & https://doi.org/10.1016/j.mechrescom.2020.103561 \\
\hline
\end{tabular}

\title{
Nonlinear bending of cylindrical shells subjected to transverse loads
}

\author{
Jue $\mathrm{ZHU}^{1,2}$, Long-yuan $\mathrm{LI}^{3 *}$ \\ ${ }^{1}$ Key Laboratory of Impact and Safety Engineering (Ningbo University) of Ministry of Education, Ningbo, China; \\ ${ }^{2}$ Joint Research Institute of Ningbo Univrrsity and Ningbo Special Equipment Inspection Institute, Ningbo, China \\ ${ }^{3}$ School of Engineering, Computing and Mathematics, University of Plymouth, Plymouth, Devon, United Kingdom \\ *Corresponding author: long-yuan.li@plymouth.ac.uk \\ Tel.:+44-1752-586 180; fax: +44-1752-586 101 \\ Accepted: July 3, 2020.
}

\begin{abstract}
This technical note presents an analytical solution of the nonlinear bending of cylindrical shells subjected to transverse bending loads, induced by the flattening of cross-section. The bending equation, representing the nonlinear relationship between beam bending curvature and externally applied load, is derived on the framework of a flattened cylindrical shell. Numerical examples of cylindrical-shell beams subjected to pure bending and uniformly distributed transverse bending loads are provided to demonstrate the rationality of the present analytical model.
\end{abstract}

(C) 2020 The Authors. Published by Elsevier Ltd.

Keywords: Cylindrical shell; nonlinear bending; overlization; cross-sectional flattening; beam.

\section{Introduction}

Circular hollow cylinders are widely used as the structural member in building construction, aerospace engineering, and oil industry. For a long circular hollow cylinder that is subjected to a transverse loading the member behaves not only like a hollow-section beam having flexural deformation, but also like a cylindrical shell exhibiting local cross-sectional ovalization deformation. The ovalization of cylindrical shells when subjected to bending loading was reported first by Brazier [1] in 1927. Brazier found that when a cylindrical shell was bent uniformly, it also flattened its cross-section. Following Brazier's pioneer's work, Reissner [2], Fabian [3], Aksel'rad [4], Aksel'rad and Emmerling [5], Libai and Bert [6], Tatting et al. [7], Li and Kettle [8] also investigated the nonlinear bending behaviour of the cylindrical shells induced by the cross-sectional ovalization. Moreover, Li [9], Rotter et al. [10], Xu et al. [11], Coman [12], Fajuyitan et al. [13], and Karamanos [14] investigated the flattening-induced instability problems of cylindrical shells. Recently, Sato and Ishiwata [15] analyzed the nonlinear response and corresponding Brazier limit-moment of single- and double-walled elastic tubes under pure bending. More recently, Luongo et al. [16] present an analytical study on the Brazier effect of elastic pipe beams with foam cores. The model was validated using finite element analysis results.

It is worth mentioning that the cross-sectional flattening also exists in thin-walled open section beams. Yuan et al. $[17,18]$ reported the cross-sectional flattening phenomenon in angle- and channel-section beams.

Currently, there are two approaches that are commonly used to deal with the cross-sectional ovalization of cylindrical shells. One is based on Brazier's original assumption of a two-stage deformation process, in which the overall bending deformation of the member was calculated based on flattened cross-section, whereas the influence of it on the crosssectional flattening was considered by using the minimum potential energy principle. The other is to employ more comprehensive nonlinear shell equations. The former is applicable only to some special loading cases, whereas the latter involves nonlinear equations that are too complicated to solve. In this paper, a simple approach is presented to deal with the nonlinear bending problem of cylindrical-shell beams induced by the cross-sectional ovalization when the beam is subjected to bending loads. 


\section{Cross-sectional flattening model}

When the cylindrical-shell beam is bent, the part above the neutral axis is in compression and the part below the neutral axis is in tension (see Fig.1a). The flattening force generated by the combined actions of compressive and tensile stresses on a cylindrical shell element with length $d s$ is shown in Fig.1b. On the deformed framework the bending stress (compressive or tensile stress) on each side of the deformed element can be decomposed into horizontal and vertical components as shown in Fig.1b. The horizontal components on the two sides are self-balanced; whereas the vertical components create a resultant flattening force, which, for example, in the compression zone can be expressed as follows,

$p d s=2 \sigma_{c} t \sin \left(\frac{d \theta}{2}\right) \approx \sigma_{c} t d \theta$

where $p$ is the flattening force per unit surface area, $\sigma_{c}$ is the compressive stress, $t$ is the thickness of the cylindrical shell, $d \theta$ and $d s$ are the angle and arc length of the deformed element, and $d \theta / d s$ represents the curvature of the deformed element.

(a)

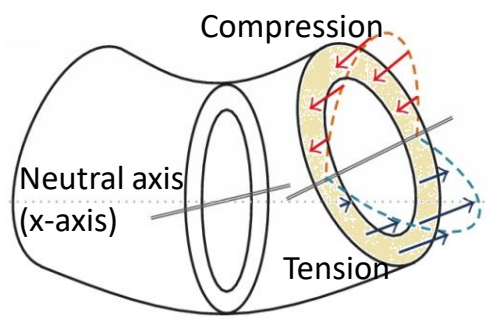

(b)

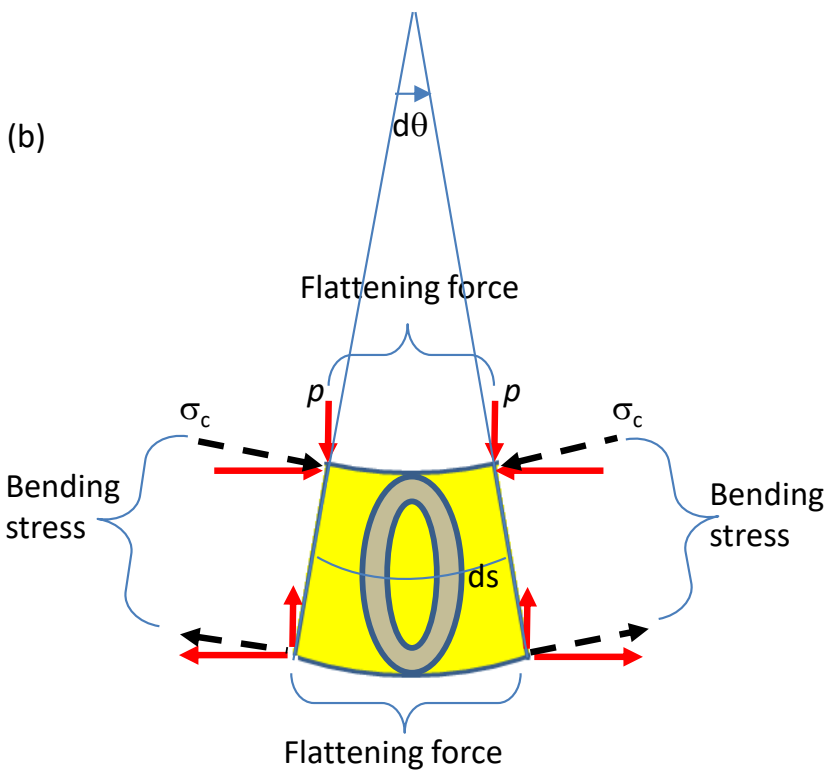

Fig.1 Flattening forces generated by bending stresses in cylindrical shells

Assume the beam-like bending deflection of the cylindrical-shell beam is $v(x)$ where $\mathrm{x}$ is the longitudinal axis of the beam. The curvature of the beam can be expressed as $d \theta / d s=d^{2} v / d x^{2}$. Thus, the flattening force can be expressed as follows,

$p=\sigma_{c} t \frac{d^{2} v}{d x^{2}}=\frac{M y t}{I} \frac{d^{2} v}{d x^{2}}$

where $M$ is the bending moment, $I$ is the second moment of area of the cylindrical-shell beam, and $y$ is the vertical coordinate from the neutral axis. By using the bending equation of Euler-Bernoulli beams, $M=E I d^{2} v / d x^{2}$, where $E$ is the Young's modulus, Eq.(2) can be rewritten as follows,

$p=\operatorname{Ety}\left(\frac{d^{2} v}{d x^{2}}\right)^{2}$

Eq.(3) indicates that the flattening force is a quadratic function of the deflection function (or curvature) and it increases with the distance away from the neutral axis. The maximum flattening force occurs at the top or bottom of the cross-section where $y$ equals to the radius of the shell with the value,

$p_{o}=E t R\left(\frac{d^{2} v}{d x^{2}}\right)^{2}$

where $p_{o}$ is the maximum flattening force and $R$ is the average radius of the cylindrical shell.

The ovalization of the cylindrical shell under the action of the distributed flattening force given by Eq.(3) can be calculated based on the curve beam model shown in Fig.2, in which the flattening force can be expressed as $p(\theta)=p_{o} \cos \theta$. According to the symmetrical conditions of the cross-section, the boundary conditions of the section at points A and B can be represented by the sliding condition without rotation as shown in Fig.2b. In addition, according to the force equilibrium condition in horizontal direction, the normal reaction force at point $\mathrm{A}$ is zero. Assume the reaction moment at point $\mathrm{A}$ is $M_{o}$, then the moment at a place with an angle $\theta$ due to the combined actions of $M_{o}$ and varying distribution load $p(\varphi)$ where $0<\varphi<\theta$ can be expressed as follows,

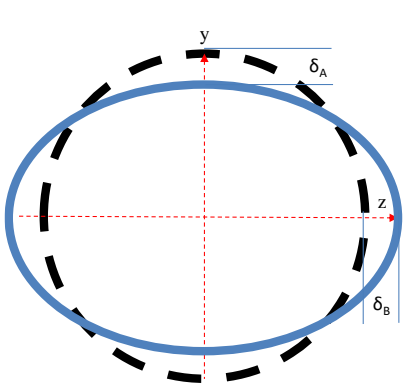

(a)

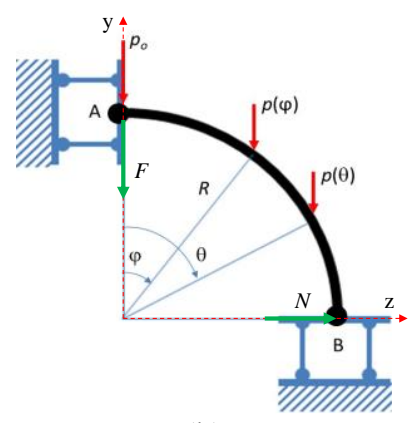

(b)
Fig.2 Model used to calculate ovalization of cylindrical shells

$$
\begin{aligned}
M(\theta) & =M_{o}-\int_{o}^{\theta}\left(p_{o} \cos \varphi\right)(R \sin \theta-R \sin \varphi) R d \varphi \\
& =M_{o}-\frac{1}{2} p_{o} R^{2} \sin ^{2} \theta
\end{aligned}
$$

The reaction moment $M_{o}$ can be calculated by using the Castigliano's theorem. The bending strain energy of the quarter of the cross-section per unit length along the $\mathrm{x}$-axis can be expressed as follows,

$U=\frac{1}{2 D} \int_{o}^{\frac{\pi}{2}} M^{2}(\theta) R d \theta$ 


$$
=\frac{1}{2 D} \int_{o}^{\pi / 2}\left(M_{o}-\frac{1}{2} p_{o} R^{2} \sin ^{2} \theta\right)^{2} R d \theta
$$

where $D=E t^{3} /\left[12\left(1-v^{2}\right)\right]$ is the wall-thickness bending rigidity of the cylindrical shell and $v$ is the Poisson ratio. The application of the Castigliano's theorem leads,

$\frac{\partial U}{\partial M_{o}}=\frac{1}{D} \int_{o}^{\pi / 2}\left(M_{o}-\frac{1}{2} p_{o} R^{2} \sin ^{2} \theta\right) R d \theta=0$

This gives the following result,

$M_{o}=\frac{1}{4} p_{o} R^{2}$

Assume there is a dummy vertical force $F$ applied at point A (Fig.2b) in the same direction as $p_{o}$. In this case the moment at any angle $\theta$ caused by $M_{o}, F$, and varying distribution load $p(\varphi)$ where $0<\varphi<\theta$ can be expressed as follows,

$M(\theta)=M_{o}-\frac{1}{2} p_{o} R^{2} \sin ^{2} \theta-F R \sin \theta$

The corresponding bending strain energy of the quarter of the cross-section can be expressed as follows,

$$
\begin{aligned}
U & =\frac{1}{2 D} \int_{o}^{\frac{\pi}{2}} M^{2}(\theta) R d \theta \\
& =\frac{1}{2 D} \int_{o}^{\pi / 2}\left(M_{o}-\frac{1}{2} p_{o} R^{2} \sin ^{2} \theta-F R \sin \theta\right)^{2} R d \theta
\end{aligned}
$$

The vertical deflection of the section at point A thus can be obtained as follows,

$$
\begin{aligned}
\delta_{A} & =\left.\frac{\partial U}{\partial F}\right|_{F=0}=\frac{1}{D} \int_{o}^{\frac{\pi}{2}}\left(M_{o}-\frac{1}{2} p_{o} R^{2} \sin ^{2} \theta\right)(-R \sin \theta) R d \theta \\
& =\frac{p_{o} R^{4}}{12 D}
\end{aligned}
$$

Similarly, assume there is a dummy horizontal force $N$ applied at point B (Fig.2b) in the z-direction. In this case the moment at any angle $\theta$ can be calculated as follows,

$M(\theta)=M_{o}-\frac{1}{2} p_{o} R^{2} \sin ^{2} \theta-N R(1-\cos \theta)$

The corresponding bending strain energy of the quarter of the cross-section can be expressed as follows,

$$
\begin{aligned}
U & =\frac{1}{2 D} \int_{o}^{\frac{\pi}{2}} M^{2}(\theta) R d \theta \\
& =\frac{1}{2 D} \int_{o}^{\pi / 2}\left[M_{o}-\frac{1}{2} p_{o} R^{2} \sin ^{2} \theta-N R(1-\cos \theta)\right]^{2} R d \theta
\end{aligned}
$$

The horizontal deflection of the section at point B thus can be obtained as follows,

$$
\begin{aligned}
\delta_{B} & =\left.\frac{\partial U}{\partial N}\right|_{N=0}=\frac{1}{D} \int_{o}^{\frac{\pi}{2}}\left(M_{o}-\frac{1}{2} p_{o} R^{2} \sin ^{2} \theta\right) R(\cos \theta-1) R d \theta \\
& =\frac{p_{o} R^{4}}{12 D}
\end{aligned}
$$

Substituting Eq.(4) into Eqs.(11) and (14), it yields,

$$
\delta=\delta_{A}=\delta_{B}=\left(1-v^{2}\right)\left(\frac{R}{t}\right)^{2}\left(\frac{d^{2} v}{d x^{2}}\right)^{2} R^{3}
$$

Eq.(15) indicates that, due to the cross-sectional ovalization the cylindrical shell becomes an elliptical shell with a long axis radius $(R+\delta)$ and a short axis radius $(R-\delta)$. The ovalization deformation is proportional to the square of bending curvature of the cylindrical-shell beam. It should be mentioned herein that Eq.(15) is derived based on the initial geometrical configuration of the cylindrical shell. This means that the higher order terms related to $\delta$ could have been neglected. Thus, strictly speaking, the accuracy of Eq.(15) is only to the order of $\mathrm{O}\left(\mathrm{d}^{2} \mathrm{v} / \mathrm{dx}^{2}\right)^{2}$. Indeed, if the approximation is only taken to the order of $\mathrm{O}\left(\mathrm{d}^{2} \mathrm{v} / \mathrm{dx}^{2}\right)^{2}$, then Eq.(15) is the same as those derived using the energy methods $[9,16]$.

\section{Overall bending stiffness of flattened cylindrical-shell beam}

The cross-sectional flattening of the cylindrical shell leads to a reduction of the overall bending stiffness of the cylindrical-shell beam. The second moment of area of the flattened cylindrical-shell beam can be calculated as follows,

$$
\begin{aligned}
& I=4 t \int_{o}^{\frac{\pi}{2}} y^{2} \sqrt{\left(\frac{d y}{d \theta}\right)^{2}+\left(\frac{d z}{d \theta}\right)^{2}} d \theta \\
& =4 t(R-\delta)^{3} \int_{o}^{\frac{\pi}{2}} \cos ^{2} \theta \sqrt{\sin ^{2} \theta+\left(\frac{R+\delta}{R-\delta}\right)^{2} \cos ^{2} \theta} d \theta
\end{aligned}
$$

where $y=(R-\delta) \cos \theta$ and $z=(R+\delta) \sin \theta$ are the two principal axes of the elliptical shell, respectively. Note that, for the case where $\delta<<R$ the following approximation can be taken,

$$
\begin{gathered}
\sqrt{\sin ^{2} \theta+\left(\frac{R+\delta}{R-\delta}\right)^{2} \cos ^{2} \theta}=\sqrt{1+\frac{4 R \delta}{(R-\delta)^{2}} \cos ^{2} \theta} \\
\approx 1+\frac{2 R \delta}{(R-\delta)^{2}} \cos ^{2} \theta-\frac{2 R^{2} \delta^{2}}{(R-\delta)^{4}} \cos ^{4} \theta
\end{gathered}
$$

Substituting Eq.(17) into (16), it yields,

$$
\begin{aligned}
I & \approx \pi t(R-\delta)^{3}\left(1+\frac{3 R \delta}{2(R-\delta)^{2}}-\frac{5 R^{2} \delta^{2}}{4(R-\delta)^{4}}\right) \\
& \approx I_{o}\left[1-\frac{3}{2} \frac{\delta}{R}+\frac{1}{4}\left(\frac{\delta}{R}\right)^{2}\right]
\end{aligned}
$$

where $I_{o}=\pi t R^{3}$ is the second moment of area of the cylindrical shell with no flattening. Substituting Eq.(15) into (18), it yields,

$I \approx I_{o}\left[1-\frac{3\left(1-v^{2}\right)}{2}\left(\frac{R^{2}}{t} \frac{d^{2} v}{d x^{2}}\right)^{2}+\frac{\left(1-v^{2}\right)^{2}}{4}\left(\frac{R^{2}}{t} \frac{d^{2} v}{d x^{2}}\right)^{4}\right]$

Note that if an oval shape with zero membrane strain is used for calculating the second moment of area of the flattened cylindrical shell $[9,16]$, then the coefficient of the $3^{\text {rd }}$ term in the brackets of Eq.(19) would be 5/8, instead of $1 / 4$. Nevertheless, the elliptical and oval shapes are very similar, particularly before the beam has a limit point instability where the ratio of $\delta / R$ is only about $2 / 9$ [9], indicating that the higher order term of $\delta^{2}$ or $\left(\mathrm{d}^{2} \mathrm{v} / \mathrm{dx}^{2}\right)^{4}$ has little influence on the overall nonlinear bending behaviour of the beam. 


\section{Nonlinear bending of cylindrical-shell beams subjected to transverse loadings}

For beams subjected to transverse loadings, the relationship between the bending moment and bending deflection when the cross-sectional flattening is considered can be expressed as follows,

$E I \frac{d^{2} v}{d x^{2}}=M_{q}$

where $M_{q}$ is the internal bending moment of the beam. Substituting Eq.(19) into (20), it yields,

$E I_{o}\left[1-\frac{3\left(1-v^{2}\right)}{2}\left(\frac{R^{2}}{t} \frac{d^{2} v}{d x^{2}}\right)^{2}+\frac{\left(1-v^{2}\right)^{2}}{4}\left(\frac{R^{2}}{t} \frac{d^{2} v}{d x^{2}}\right)^{4}\right] \frac{d^{2} v}{d x^{2}}=M_{q}$

Eq.(21) is the nonlinear bending equation of the cylindricalshell beam with considering the cross-sectional flattening. It is obvious that for a given externally applied load, $M_{q}$ is known, and thus the curvature and the corresponding deflection function can be determined by solving the nonlinear equation Eq.(21).

As the examples, herein we first consider a beam subjected to a pure bending moment $M_{o}$. Hence, $M_{q}=M_{o}$ is constant and Eq.(21) becomes,

$$
\left[1-\frac{3\left(1-v^{2}\right)}{2}\left(\frac{R^{2}}{t} \frac{d^{2} v}{d x^{2}}\right)^{2}+\frac{\left(1-v^{2}\right)^{2}}{4}\left(\frac{R^{2}}{t} \frac{d^{2} v}{d x^{2}}\right)^{4}\right]\left(\frac{R^{2}}{t} \frac{d^{2} v}{d x^{2}}\right)=\frac{M_{O}}{\pi t^{2} R E}
$$

For a given $M_{o}$, one can algebraically solve Eq.(22) for obtaining the curvature. Fig.3 graphically shows the variation of the bending curvature with the applied moment obtained numerically from Eq.(22). It can be seen from the figure that when the dimensionless applied moment $\left(M_{o} /\left(\pi t^{2} R E\right)\right.$ is smaller than 0.2 , the dimensionless curvature $\left(R^{2} / t\right) d^{2} v / d x^{2}$ is almost linear with the applied moment. However, when $M_{o} /\left(\pi t^{2} R E\right)>0.2$ the nonlinearity between the dimensionless applied moment and dimensionless curvature becomes very obvious. The beam has a limit point instability at the point $(0.524,0.336)$, which are very close to $(0.494,0.329)$ and $(0.524,0.333)$ reported in [9] and [16], respectively. The two nonlinear solutions in which the second moment of area of the flattened cylinder is calculated based on elliptical and oval shape are very close before the beam has the limit point instability. The difference between them becomes obvious only after their limit points.

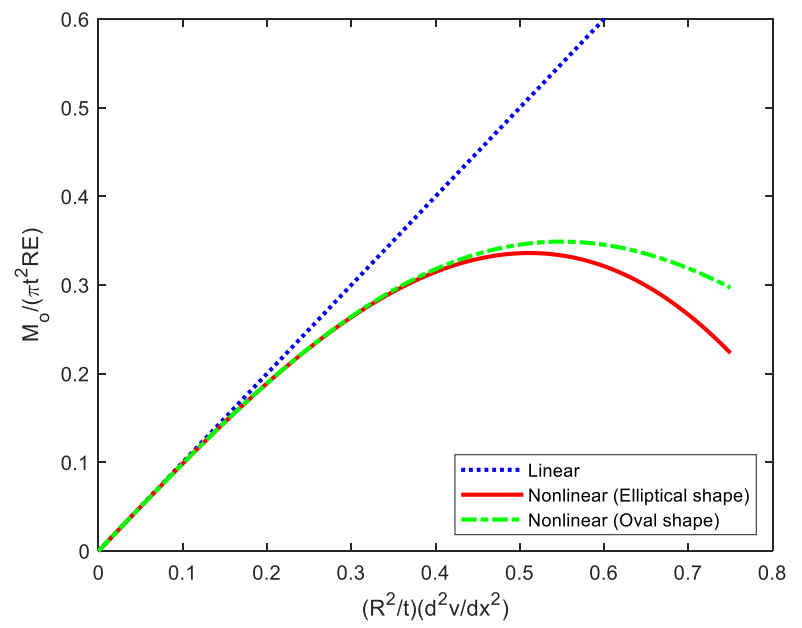

Fig.3 Linear and nonlinear solutions of the beam with pure bending $(v=0.3)$.

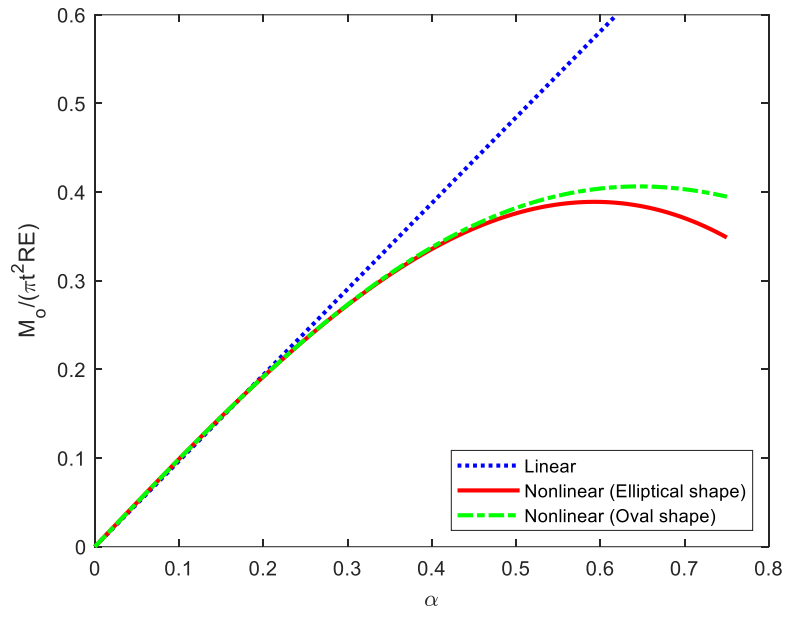

Fig.4 Linear and nonlinear solutions of the beam with uniformly distributed load $(v=0.3)$.

The second example is the simply supported beam with a uniformly distributed transverse load $q$. In this case the internal bending moment is $M_{q}=4 M_{o}\left(\xi-\xi^{2}\right)$ where $M_{o}=q l^{2} / 8$ is the largest moment and $\xi=x / l$ the dimensionless axial coordinate, and the nonlinear bending equation Eq.(21) is expressed as follows,

$$
\begin{aligned}
& {\left[1-\frac{3\left(1-v^{2}\right)}{2}\left(\frac{R^{2}}{t l^{2}} \frac{d^{2} v}{d \xi^{2}}\right)^{2}+\frac{\left(1-v^{2}\right)^{2}}{4}\left(\frac{R^{2}}{t l^{2}} \frac{d^{2} v}{d \xi^{2}}\right)^{4}\right]\left(\frac{R^{2}}{t l^{2}} \frac{d^{2} v}{d \xi^{2}}\right)=} \\
& \frac{4 M_{O}\left(\xi-\xi^{2}\right)}{\pi t^{2} R E}
\end{aligned}
$$

Eq.(23) is a nonlinear differential equation and there is no exact solution available in literature. For obtaining an approximate solution of the beam with simply supported boundary conditions, we assume the solution of Eq.(23) can be expressed as follows,

$v(\xi)=-\frac{t l^{2}}{(\pi R)^{2}} \alpha \sin \pi \xi$

Substituting Eq.(24) into (23) and using Galerkin method, we have

$\int_{o}^{1}\left[1-\frac{3\left(1-v^{2}\right)}{2}(\alpha \sin \pi \xi)^{2}+\right.$

$\left.\frac{\left(1-v^{2}\right)^{2}}{4}(\alpha \sin \pi \xi)^{4}\right]\left(\alpha \sin ^{2} \pi \xi\right) d \xi=\int_{o}^{1} \frac{4 M_{o}\left(\xi-\xi^{2}\right)}{\pi t^{2} R E} \sin \pi \xi d \xi$

The integration of Eq.(25) leads to

$\left(1-\frac{3\left(1-v^{2}\right)}{2} \frac{3}{4} \alpha^{2}+\frac{\left(1-v^{2}\right)^{2}}{4} \frac{5}{8} \alpha^{4}\right) \frac{\alpha}{2}=\frac{M_{O}}{\pi t^{2} R E} \frac{16}{\pi^{3}}$

Fig.4 graphically shows the variation of the beam deflection at the mid-span with the applied load obtained numerically from Eq.(26). Again, when $M_{o} /\left(\pi t^{2} R E\right)>0.2$ the nonlinearity between the dimensionless applied load and dimensionless deflection becomes obvious. When $M_{o} /\left(\pi t^{2} R E\right)$ attends to 0.377 the beam has a limit point instability.

Note that the cylindrical shells discussed in the above two examples are the statically determinate cylinders in which case the bending moment can be determined statically. For statically indeterminate cylinders, however, the bending moment $\mathrm{M}_{\mathrm{q}}$ in Eq.(21) depends not only on the externally 
applied loads but also on the deflection of the cylinders. This leads the nonlinear differential equation of Eq.(21) much more complicated and its solution is much more difficult to obtain, although it is not impossible for some simple cases.

\section{Conclusions}

This paper has presented an analytical study on the nonlinear bending response of cylindrical-shell beams when subjected to bending loads induced due to cross-sectional flattening. The study has shown that the bending curvature of the beam generates the flattening force, but the relationship between the cross-sectional ovalization deformation and bending curvature is nonlinear. By using the flattened crosssection geometry a generalized nonlinear bending equation of cylindrical-shell beams with considering cross-sectional flattening has been derived. Numerical examples have been also provided to demonstrate the appropriateness and rationality of the present analytical model.

Finally, it should be mentioned herein that the nonlinear bending response of cylindrical shells investigated in the present study is restricted to only elastic cylindrical shells where the ovalization deformation is dominant and thus the results obtained from this study is applicable only to the intermediate and long elastic cylindrical shells with the bending moment and bending curvature not over pass its limit instability point due to the inclusion of limited nonlinear terms. This gives the limitations of $M_{o} /\left(\pi t^{2} R E\right)<1 / 3$ for maximum moment and $\left(\mathrm{d}^{2} v / \mathrm{d} x^{2}\right)\left(R^{2} / t\right)<1 / 2$ for maximum curvature with the maximum bending stress $\mathrm{Et} /(3 \mathrm{R})$ not exceeding the yield stress of the material.

Declaration of interests - The authors declare that they have no known competing financial interests or personal relationships that could have appeared to influence the work reported in this paper.

Acknowledgment - The first author would like to acknowledge the financial support received from National Natural Science Foundation of China (No.11572162 and No.11972203), Ningbo Rail Transit (JS-00-SG-17003), and K.C. Wong Magna Fund at Ningbo University for their financial support.

\section{References}

[1] L. G. Brazier, On the flexture of thin cylindrical shells and other thin sections. Proc. R. Soc. Ser. A 116(773) (1927) 104-11.

[2] E. Reissner, On finite bending of pressured tubes. J. Appl. Mech. (ASME) 26 (1959) 386-392.

[3] O. Fabian, Collapse of cylindrical elastic tubes under combined bending, pressure and axial loads. Int. J. Solids Struct. 13 (1977) 1257- 1270

[4] E. L. Aksel'rad, Pinpointing the upper critical bending load of a pipe by calculating geometric nonlinearity. Izv. Akad. Nauk SSR Mekh. 4 (1965) $133-139$.

[5] E. L. Aksel'rad, F. A. Emmerling, Collapse load of elastic tubes under bending. Isr. J. Technol. 22 (1984) 89-94.

[6] A. Libai, C. W. Bert, A mixed variational principle and its application to the nonlinear bending problem of orthotropic tubes-II. Application to nonlinear bending of circular cylindrical tubes. Int. J. Solids Struct. 31 (1994) 1019-1033.

[7] B. F. Tatting, Z. Gurdal, V. V. Vasiliev, The Brazier effect for finite length composite cylinders under bending. Int. J. Solids Struct. 34 (1997) 1419-1440.

[8] L. Y. Li, R. Kettle, Nonlinear bending response and buckling of ringstiffened cylindrical shells under pure bending. International Journal of Solids and Structures 39 (2002) 765-781.

[9] L. Y. Li, Bending instability of composite tubes. J. Aerospace Eng. (ASCE) 9 (1996) 59-61.

[10] M. J. Rotter, S. Michael, J. Adam, C. Lei, Nonlinear stability of thin elastic cylinders of different length under global bending. International Journal of Solids and Structures 51 (15-16) (2014) 2826-2839.

[11] Z. Y. Xu, L. Gardner, A. J. Sadowski, Nonlinear stability of elastic elliptical cylindrical shells under uniform bending. International Journal of Mechanical Sciences 128-129 (2017) 593-606.

[12] C. D. Coman, Bifurcation instabilities in finite bending of circular cylindrical shells. International Journal of Engineering Science 119 (2017) 249-264.

[13] O. K. Fajuyitan, A. J. Sadowski, M. A. Wadee, J. M. Rotter, Nonlinear behaviour of short elastic cylindrical shells under global bending. ThinWalled Structures 124 (2018) 574-587.

[14] S. Karamanos, Bending instabilities of elastic tubes, Int. J. Solids Struct. 39 (8) (2002) 2059-2085..

[15] M. Sato, Y. Ishiwata, Brazier effect of single- and double-walled elastic tubes under pure bending, Struct. Eng. Mech. 53 (1) (2015) 17-26.

[16] A. Luongo, D. Zulli, I. Scognamiglio, The Brazier effect for elastic pipe beams with foam cores. Thin-Walled Structures 124 (2018) 72-80.

[17] W. B. Yuan, Geometric nonlinear bending response and buckling of angle-section beams under pure bending. Journal of Constructional Steel Research 85 (2013) 73-80.

[18] W. B. Yuan, Nonlinear instability analyses of channel-section beams subjected to minor-axis pure bending. International Journal of Mechanical Sciences 73 (2013) 77-81. 